\title{
The investigation of the solar active regions structure using the multiwave observations with Large Solar Coronagraph of Sayan Observatory: the study of spectra and images of the sunspot umbras in the domain of the line He I $10830 \AA$
}

\author{
S. A. Chuprakov, G. I. Kushtal, P. G. Papushev, V. I. Skomorovsky, \\ Yu. S. Zagaynova
}

Institute for Solar-Terrestrial Physics, Russia, 664033, Irkutsk, Lermontova str. 126, email: chupr@iszf.irk.ru

\begin{abstract}
The solar active regions observations were carried out with facilities for multi-spectral investigations of solar atmosphere on Large solar coronagraph of Sayan observatory. The facilities permits to perform the spectral observations in selected chromospheric and coronal lines simultaneously with obtaining of $H_{\alpha}$ and He I images.

In this work we analyze the results of spectral observations in the range from $10824 \AA$ to $10835 \AA$ (the He I $10830 \AA$ and Si I $10827 \AA$ lines) and images of the sunspot umbrae in the He I $10830 \AA$.
\end{abstract}

\section{Introduction}

The approaches which provide simultaneous data acquisition in a wide range of thermodynamic conditions with the high spatial and time resolution are particularly interesting among the methods of multiwave solar astrophysics. The acquisition of the quantitative characteristics of the fine structure of solar atmosphere is a particularly difficult experimental problem due to the extremely small characteristic sizes of the events. The ground-based observations with the high spatial, spectral and temporal resolution compare well with the space- born observations and significantly complete them.

The observation at the visual and near infrared range (NIR) of the spectrum bears an advantage based on the fact that the lines of the most widespread chemical elements, with sufficiently low potentials of excitation are located in this range. The investigations of these lines with the help of spectral equipment of high resolution and narrow-band tunable filters form the base of modern knowledge about the structure of solar atmosphere and serve as a reference frame for the analysis of phenomena detected by the space observatories.

We have created the setup for the multiwave observation at the 53-cm non-eclipse coronagraph of Sayan mountain observatory. This telescope is known as Nicolsky coronagraph (NC). Due to the simple and reliable optical system NC was successfully applied to observations of solar corona as well as for research of the fine structure of chromosphere.

The NC, established at Sayan observatory was essentially modernized at the last years. Its mechanical units and electronics were completely changed. Replacement of the tape drive of its axes by the more exact worm gear mechanism in combination with the automated control system provides an pointing error of about $0.5-1$ arc. sec. Equipment 
of the coronagraph by the automated control system on based of the local microprocessor network provides functions of pointing, guiding and focussing of an objective and CCD detectors. It allowed creating the complete the management complex which meets the requirements of the modern observational tasks. Practically all numerous units of coronagraph were upgraded, but its unique optical system was left without changes.

Other important innovation in the equipment of the coronagraph was an installation of two-bandpass birefriengent tunable filter (TBBF) which can give images on the hydrogen line $6563 \AA\left(\mathrm{H}_{\alpha}\right.$ line) and infrared He I with passband of about 0.46 and $0.3 \AA$, respectively (V. I. Skomorovsky et al. 2000)

It is impossible to perform the simultaneous observations in the various spectral lines in the traditional sense because of significant chromatism. However, it is possible to realize a mode of simultaneous observation of structure and spectra of photosphere, chromosphere and corona (at the case of the limb observation) using the combined spectral-filter observations in various combinations of spectral lines and monochromatic images. We realized the mode of multiwave observation at the coronagraph in the following spectral ranges: SiI $10827 \AA$, He I 10829-10830 A, Fe XIII 10747-10798 $\AA$ - spectrograph and He I - $\mathrm{H}_{\alpha}$ $6563 \AA$ - TBBF.

The EEV $2048 \times 2048$ CCD chip with $24 \mathrm{mkm}$ pixel was utilized for registration of the spectra. The ST-133 CCD camera controller manufactured by Princeton Instruments, Inc. has been applied for high speed (up to 1 megapixel per second) and high performance 16 bit image acquisition. The time of expose at NIR is about 1 sec. The TEK $512 \times 512$ and THOMSON $1024 \times 1024$ CCD with 16 bit data acquisition controllers has been utilized for $\mathrm{H}_{\alpha}$ and $\mathrm{He} \mathrm{I}$ images registration.

\section{Data preprocessing line profiles analysis}

Images in the He I line have a low contrast. Filtergram data have been preprocessed with standard procedures - background subtraction and flatfield division. The line profile analysis of He I line is complicated. The presence of blend lines influences on quality of continuum correction, therefore it is necessary to select the wavelength interval which is free from lines. Values of a continuum gets out in big enough interval $( \pm 7 \AA)$ and in those areas, where a continuous spectrum is free from blends lines (D. Hall et al. (1973)), due to an opportunity of registration of the big spectral region. In our investigation we use the wavelehgth range 10823.0371-10837.0935 $\AA$ for holding the continuum level.

Two He I components are well resolved after elimination of the nearest lines only. We use Voigt function for approximation of the nearest blended lines Si I 10827.09 $\AA$ and $\mathrm{H}_{2} \mathrm{O}$ $10832.09 \AA$. It is difficult to approximate these lines well, because there are many slightly blend lines in their wings and the Si I line is magnetic sensitives. Umbrae spectrum, which we investigated, contain additional features as weak molecular depressions. To resolve all these problems, we select blend free intervals in approximated line profile. Further, these lines have being extrapolated and subtracted along whole investigated spectral region, where the residual intensity rises to $r_{\lambda}=1.0 \pm 0.01$.

For profile analysis of the He I $10829 \AA$ and $10830 \AA$ lines were approximated by two Gauss functions separately, which parameters are accepted for result of processing. Residual mistakes at behaviour of a continuous spectrum do not exceed $0.3 \%$.

\section{Results of spectral-filter active region observation in $\mathbf{H e ~ I ~}$}

The infrared helium line was intensively studied at last years and there is a full phenomenological picture of observably structures and the phenomena was provided. The clear physical mechanism of this line formation, nevertheless, does not exist. Recently models of formation and radiation transfer as a whole correctly describe of the line 


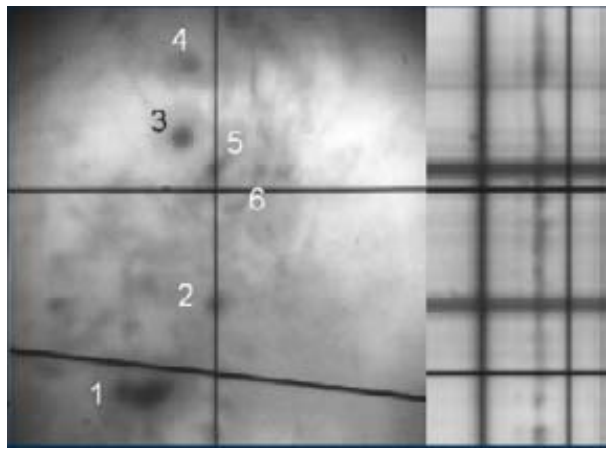

Figure 1. Samples of the NOAA AR 0397, taken july 42003 09:28 UT, features, He I line parameters for which are given in the tables later. Vertical line on the image - spectrograph entrance slit, horizontal lines - scale threads. On the left of the image - spectergram for the same region, taken quasi-simultaneously with image.

$\begin{array}{lccccccccc}\text { Date } & \text { Local time } & \text { NOAA AR features } & \mathrm{S}, \mathrm{mSH} & \mathrm{r}_{\lambda} & \Delta \lambda_{D}, \AA & \mathrm{W}_{\lambda}, \AA & \phi \\ \text { 14.09.2002 } & & 0105 & & 106 & 0.1297 & 0.6673 & 0.2171 & 0.4 \\ 04.07 .2003 & 09: 11-09: 48 & 0397 & 1 & 82.3 & 0.1011 & 0.6946 & 0.176 & 0.34 \\ 04.07 .2003 & 10: 56-12: 36 & 0397 & 3 & 36.3 & 0.1274 & 0.6365 & 0.2033 & 0.46 \\ 04.07 .2003 & 18: 28-19: 21 & 0397 & 5 & 31.2 & 0.1524 & 0.6335 & 0.2421 & 0.48 \\ 04.07 .2003 & 19: 22-19: 48 & 0397 & 4 & 19.1 & 0.1612 & 0.6236 & 0.2519 & 0.56 \\ 04.07 .2003 & 09: 50-10: 54 & 0397 & 2 & 15.4 & 0.1672 & 0.619 & 0.2593 & 0.68 \\ 06.07 .2003 & & 0400 & & 13.6 & 0.1652 & 0.5835 & 0.242 & 0.69 \\ 06.07 .2003 & & 0400 & & 10.2 & 0.1934 & 0.5964 & 0.289 & 0.65 \\ 06.07 .2003 & & 0400 & & 8.8 & 0.1745 & 0.592 & 0.2589 & 0.72 \\ 04.07 .2003 & 19: 05-19: 18 & 0397 & 6 & 7.1 & 0.2284 & 0.5839 & 0.3344 & 0.7\end{array}$

Table 1. Active region numbers and He I main component profile parameters for umbras, shown on picture below. Numbers of the umbras in "features" column corresponded to labels on the image.

behaviors in the large scales, but are poorly suitable for understanding of the fine structure phenomena. The study of morphology of formation of absorption of He I line on the sun disk point to, that an absorption become stronger at the presence of a magnetic field. This line is observed by the deepest in plages, where a magnetic field basically perpendicularly to a line of sight. At the longitudinal magnetic field absorption also strengthen. However details of behaviour of a line in various magnetic features were investigated insufficiently. Among numerous of the works devoted to studying of an absorption He I line on a disk of the Sun, only few was devoted to study of this line profile at sunspot umbra.

The observation of some AR in He I line was carried out in 2002-2003. Observation of NOAA AR 0397 at 4.07.2003 and 0105 at 14.09.2002 were utilized to study in every detail He I line profiles at sunspots and its umbrae. Figure 1 show a sample spectral and filtergram frames at He I line. The studies AR were placed near disc center. In the table 1 average values parameters of the He I line profiles and value of contrast of the umbrae relative to photospheric continuum at this range are given. The data in the table are ordered depending on the total area of the umbrae, determined with the help the continuum data received simultaneously from the TRACE spacecraft.

The values of central line depth $r_{\lambda}$ and equivalent width $W_{\lambda}$ as determined by the above analysis for sunspot umbrae shown in table 1 are similar those which was findings 
at early papers T. Fay et al. (1972), P. G. Papushev et al. (1979). Distinguish from the present work is, that the results are based on the bulk experimental data. Furthermore TBBF data allow to determined position of a spectral slit more precisely. These additional opportunities allow estimating parameters of the line profiles with error not more than several percents. The achieved accuracy allows counting as real distinctions in Doppler halfwith $10829 \AA$ and $10830 \AA$ lines, equal to 10-20\% and showing the weak tendency of growth with increase of the sunspot area. The average equivalent width of strong and weak components of He I triplet in spots umbrae are in the ratio of $4.6 \pm 1.15$. New observation allow to confirm differences of the He I line profiles in an umbra both environmental penumbras and plages. At the same time the increases of $\mathrm{W}_{\lambda}$ depending on umbrae's area noticed in early works does not find full confirmation according to the new data.

For an establish of this dependence as well as for to account for scattered light influences effect on to parameters of a line above mentioned, the precision technique of the account of scattered light is necessary. Our data allow to determine the amount of the scattered light on the basis of measurements of contrast of a umbrae at continuum wavelength. The data on value of the umbra-continuum contrast $\phi$ are given in the literature (C. W. Allen (1973), V. N. Obridko (1985)). However, it was measured at sunspots with the characteristic size of an umbrae equal to 40-60 arc. sec. The true value of contrast near $10830 \AA$ continuum wavelength is equal to $0.3-0.33$. It is accepted, that contrast true values does not depend on umbrae's sizes. However, we do not have justificationbased data to believe, that this statement is true proposition for spots with the sizes less than 10 arc. sec. and pores. The main part of the spots umbrae's were investigated in our observation had a sizes less than 20 arc.sec. (about $100 \mathrm{mSH}$ ). For largest of them observably contrast is close to true values. Also it is possible to present a number of indirect evidences that occurrence of absorption of He I line in the small sizes magnetic structures is not a methodical artifact. The more detailed consideration which is taking into account both various sources of scattered light, and new measurements of true contrast of small spots and pores must be carry out. Because of at this paper we shall be limited to represent only row data.

\section{Acknowledgements}

Authors thank A.P.Kurbaka, E.V.Klunko, V.I.Tergoev, G.N.Domyshev and V.P.Sadohin for technical support. One of us (PP) is especially grateful prof. V.Grigor'ev for granting of three-day administrative holiday and discussion about of the account of a scattered light and calibration of the data, prof. R.B.Teplitskaya and dr. E.S.Kulaginu for useful discussions of the questions mentioned in work.

The work was supported in part by grant RFFI 98-02-11 and the program of the Ministry of a Science and Education of the Russian Federation "Astronomy".

\section{References}

T. Fay, A. Wyller and H. Yan 1972 Solar Phys. 23, 58-77.

Donald N. B. Hall and James B. Breckinridge 1973 Solar Phys., 28, 15-20

C. W. Allen 1973 Univers. of London

P. G. Papushev and O. A. Borodina 1979 Sov. Astron. Lett. 5, 620-621.

V. N. Obridko 1985 Moscow, "Nauka"

V. I. Skomorovsky, G. I. Kushtal 2000 Opt. Zhurn. 67, 99-107. 